\title{
X-ray reflectivity studies of atomic-level surface-segregation in a liquid eutectic alloy of AuSn
}

\author{
Venkatachalapathy S. K. Balagurusamy, ${ }^{1}$ Reinhard Streitel, ${ }^{1}$ Oleg G. Shpyrko, ${ }^{2}$ P. S. Pershan, ${ }^{1}$ Mati Meron, ${ }^{3}$ and Binhua Lin ${ }^{3}$ \\ ${ }^{1}$ Department of Physics and DEAS, Harvard University, Cambridge, Massachusetts 02138, USA \\ ${ }^{2}$ Center for Nanoscale Materials, Argonne National Laboratory, Argonne, Illinois 60439, USA \\ ${ }^{3}$ CARS, University of Chicago, Chicago, Illinois 60637, USA
}

(Received 27 November 2006; revised manuscript received 2 February 2007; published 29 March 2007)

\begin{abstract}
$\mathrm{X}$-ray reflectivity studies reveal atomic-level surface-segregation at the free surface of the eutectic $\mathrm{Au}_{71} \mathrm{Sn}_{29}$ liquid alloy. The surface-segregation extends up to three layers, in which the top layer is almost a pure monolayer of Sn, the second layer is almost a pure monolayer of Au and the third layer appears to be slightly enhanced in Au. Although the surface-segregation concentration profiles can be qualitatively accounted for by the theories of Defay-Prigogine and Strohl-King, they cannot satisfactorily account for the measured surface tension.
\end{abstract}

DOI: 10.1103/PhysRevB.75.104209

PACS number(s): 61.20.-p, 61.10.-i, 68.03.- $-\mathrm{g}$

\section{INTRODUCTION}

The Gibbs adsorption rule predicts that for liquid mixtures of two elements with disparate surface tensions the surface concentration of the component with the lower surface tension will be enhanced. ${ }^{1-3}$ This rule has been successfully applied to explain the observed segregation of the lowsurface tension component to the surface in numerous binary liquid mixtures. ${ }^{2}$ Until recently most of the studies have examined the surface-segregation behavior by measuring either adsorption isotherms or surface tension values. ${ }^{2}$ Unfortunately, these methods do not reveal the kind of details about the depth dependence of the atomic segregation near the surface that is necessary for full understanding of the interfacial electronic and thermodynamic properties. On a practical level alloys such as InSn, AuSn, and BiSn are typical of the wide class of materials that are being actively studied as substitutes for toxic Pb-based solder in electronics applications and as packaging materials with good mechanical properties and there is need for more specific information on the nature of their surfaces. ${ }^{4,5}$ Although some insights in the near surface regions of the liquid or solid interface can be obtained by transmission electron microscopy measurements of nanosize alloy particles such as those carried out by Mori and colleagues on just these alloys ${ }^{6,7}$ these techniques cannot provide the details of the atomic-level segregation near the surface layers of liquid alloys that can be obtained from $\mathrm{x}$-ray reflectivity studies of the type to be presented here. ${ }^{8-11}$

For example, x-ray studies have shown that the GaBi liquid alloy that has a large positive enthalpy of mixing, $1.05 \mathrm{~kJ} / \mathrm{mole}$ (Ref. 12) exhibits a rich surface behavior that includes surface segregation of the lower surface tension $\mathrm{Bi}$ into a monolayer followed by a thick wetting film of a Garich liquid between the Bi monolayer and the bulk alloy. ${ }^{8,9,13}$ $\mathrm{X}$-ray results from another alloy, BiSn (Ref. 11) for which the positive enthalpy of mixing is smaller, $0.105 \mathrm{~kJ} / \mathrm{mole}$ (Ref. 14) shows surface segregation in the top three layers with the top most layer very rich in $\mathrm{Bi}$, the next layer rich in Sn followed by a third Bi rich layer. In contrast the BiIn liquid alloy with negative enthalpy of mixing $-1.212 \mathrm{~kJ} /$ mole,${ }^{14}$ shows only modest surface segregation with the low surface tension $\mathrm{Bi}$ in the top monolayer. ${ }^{10} \mathrm{We}$ report here x-ray reflectivity studies of a AuSn liquid alloy that has large negative enthalpy of mixing $(-9.66 \mathrm{~kJ} / \mathrm{mole})$ (Ref. 14) and atomic-level surface segregation in the top three layers.

\section{EXPERIMENTAL METHOD}

Solid ingots of $\mathrm{Au}_{71} \mathrm{Sn}_{29}$ alloy sample (Alfa Aesar, 99.99\% purity) were placed in a UHV chamber in a Mo pan whose surface was cleaned by sputtering with $\mathrm{Ar}^{+}$ions. The UHV chamber was evacuated to $10^{-9}$ Torr and the bake out process was started. During the bake out, the walls of the UHV chamber and its components were heated gradually to $100-200{ }^{\circ} \mathrm{C}$ to desorb moisture. Following about 2 days of bake out, the chamber was cooled to room temperature. A Boraelectric heating element mounted underneath the sample pan was used to heat the sample to temperatures of the order of $330{ }^{\circ} \mathrm{C}$ to melt the sample. At this point the macroscopic native oxide, if any, as well as any possible contaminations at the surface were removed by mechanical scraping of the liquid surface with a Mo strip wiper. A clean liquid surface was finally obtained by further sputtering by an $\mathrm{Ar}^{+}$ion beam for several hours to remove the remaining microscopic surface oxide.

The x-ray reflectivity measurements from a clean liquid surface of the alloy were carried out at the liquid surface spectrometer facility at the ChemMat CARS beam line at the Advanced Photon Source in Argonne National Laboratory. The kinematics of these $\mathrm{x}$-ray measurements are illustrated in Fig. 1. X-rays with a wavelength of $\lambda=0.943 \AA$ corresponding to the wave vector $k_{\text {in }}=2 \pi / \lambda$, impinge on the horizontal liquid surface at an incident angle $\alpha$. The detector captures the outgoing ray with a wave vector $\vec{k}_{\text {out }}(\beta, \Delta \Theta)$. The specular reflectivity $R\left(q_{z}\right)$ is the ratio of the intensity of the outgoing ray to the incident intensity, when the conditions for specular reflection, $\alpha=\beta$ and $\Delta \Theta=0$ are fulfilled. In this case the surface-normal momentum transfer is $q_{z}=(4 \pi / \lambda) \sin \alpha$. The detector resolution due to a finite angle of acceptance was defined by horizontal and vertical slits mounted in front of the detector, approximately $650 \mathrm{~mm}$ from the sample. A second set of slits located approximately $200 \mathrm{~mm}$ from the 


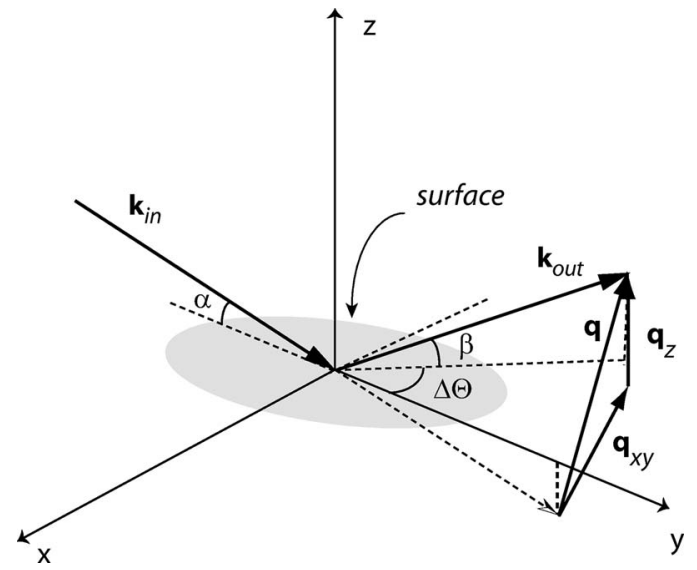

FIG. 1. Kinematics of the x-ray measurements from the sample. The incident and detected $\mathrm{x}$-ray wave vectors are $k_{\text {in }}$ and $k_{\text {out }}$, respectively.

sample on the detector arm was used to shield against unwanted parasitic scattering. During all specular reflectivity measurements the full widths of the angular acceptance of the detector were fixed at 9.4 mrad vertically and $3.1 \mathrm{mrad}$ horizontally. The measurements were carried out with the sample held at a temperature of $295^{\circ} \mathrm{C}$ with a stability better than $0.05{ }^{\circ} \mathrm{C}$. Mechanical vibrations from the liquid surface spectrometer stage were isolated from the UHV sample chamber by an active feedback-controlled vibration isolation mechanism. ${ }^{15}$ The reflected intensity was measured with an Oxford scintillation detector.

For low $q_{z}\left(<\sim 0.6 \AA^{-1}\right)$ range, the specular reflectivity is obtained by normalizing the reflected signal to the incident beam intensity. The procedure at high $q_{z}\left(>\sim 0.6 \AA^{-1}\right)$ is more complicated because of the appreciable background due to the isotropic scattering from the bulk of the liquid. This background has a broad peak centered at nearly the same $q_{z}$ as the surface-induced layering peak. Therefore, for $q_{z} \geqslant 0.6 \AA^{-1}$ a background-subtracted specular signal is represented by the difference between the signal measured at $(\alpha=\beta$ and $\Delta \Theta=0)$ and the off-specular signal that is collected at $\Delta \Theta= \pm 0.3^{\circ}(\sim 5 \mathrm{mrad})$. For $q_{z}<0.6 \AA^{-1}$ the relative contribution of the bulk liquid scattering to specular reflection is sufficiently small that it can be neglected.

The fraction of the $\mathrm{x}$-ray intensity reflected at an angle $\alpha$ from the liquid surface is given in the Born approximation $^{16,17}$

$$
R\left(q_{z}\right)=R_{F}\left(q_{z}\right) C W\left(q_{z}, T\right)\left|\Phi\left(q_{z}\right)\right|^{2}
$$

where $R_{F}\left(q_{z}\right)$ is the theoretical Fresnel x-ray reflectivity from an abrupt flat interface between vacuum and the bulk alloy. The function $C W\left(q_{z}, T\right)$ is a Debye-Waller like factor due to thermally excited capillary waves. The procedure for calculating $C W\left(q_{z}, T\right)$ has been detailed in earlier papers on surface layering in $\mathrm{Ga},{ }^{18} \mathrm{In},{ }^{16} \mathrm{~K},{ }^{17}$ and water. ${ }^{19}$ The basic idea is to convolve the known resolution with the theoretical algebraic form $\sim 1 /\left|\vec{q}_{x y}\right|^{2-\eta}$ where

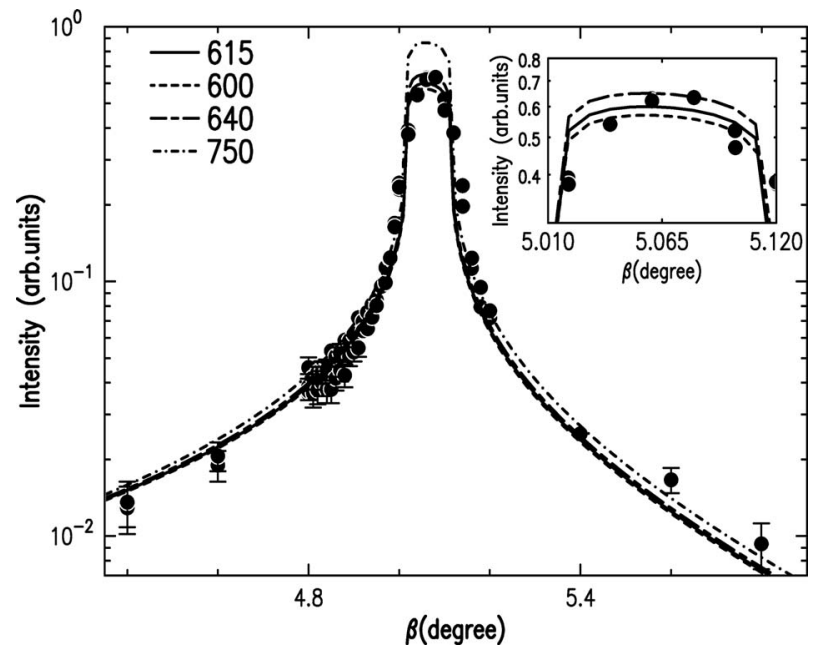

FIG. 2. Off-specular diffuse scattering from the liquid surface for the purpose of measuring the surface tension. The peak corresponds to the specular condition $\beta=5.07^{\circ}\left(q_{z}=1.1 \AA^{-1}\right)$. The profile calculated from the model is shown for the best fit value of surface tension $\gamma=615 \mathrm{mN} / \mathrm{m}(-)$. The inset is an expanded version of the best fit and confidence limits at the specular peak. Calculations shown for $\gamma=600 \mathrm{mN} / \mathrm{m}(---), \gamma=640 \mathrm{mN} / \mathrm{m} \mathrm{(--)}$ indicate the confidence limits. The line for $\gamma=750 \mathrm{mN} / \mathrm{m}$ (-.-) illustrates the calculation for average of the published values.

$$
\left|\vec{q}_{x y}\right|^{2}=(2 \pi / \lambda)^{2}\left[\cos ^{2} \alpha+\cos ^{2} \beta-2 \cos \alpha \cos \beta \cos \Delta \Theta\right]
$$

and $\eta=\left(k_{B} T / 2 \pi \gamma\right) q_{z}^{2}$ where for $\alpha \neq \beta \quad q_{z}=(2 \pi / \lambda)(\sin \alpha$ $+\sin \beta$ ). The actual form used in Eq. (1) is the difference between the convolution at $\alpha=\beta$ and $\Delta \Theta=0$ and the average of the convolution at the offset angles of $\Delta \Theta= \pm 0.3^{\circ}$.

The surface tension for the alloy can be determined from the $\eta$ dependence of the diffuse scattering from thermal capillary waves. ${ }^{17,19}$ Figure 2 contains a comparison between the background subtracted diffuse scattering data (filled circles) and the $\beta$ dependence of a background-subtracted convolution of the theoretically derived scattering cross section (solid line). The narrow detector vertical resolution of $1.55 \mathrm{mrad}$ for $\alpha=5.07^{\circ}$ applicable for both of these was typical of scans taken in order to measure the surface tension $\gamma$. Note that this resolution is considerably smaller than the vertical resolution of the detector, $9.4 \mathrm{mrad}$, that was used to measure $R\left(q_{z}\right)$. This data corresponds to $q_{z}=1.1 \AA^{-1}$ and the solid line is calculated for the value of surface tension of $615 \mathrm{mN} / \mathrm{m}$ that gives the best fit to the data. Broken lines corresponding to $\gamma=(600,640) \mathrm{mN} / \mathrm{m}$ illustrate the confidence limits of fit. The other broken line (-.-) illustrates the calculation for the mean value of the published values of the surface tension, $\gamma=750 \mathrm{mN} / \mathrm{m}^{20}$ The structure factor obtained from the measured reflectivity uses the value $\gamma$ $=615 \mathrm{mN} / \mathrm{m}$.

The surface structure factor, $\Phi\left(q_{z}\right)$ is given by the Fourier transform of the surface-normal derivative of the electron density $\rho(z)$ (Refs. 21 and 22) 


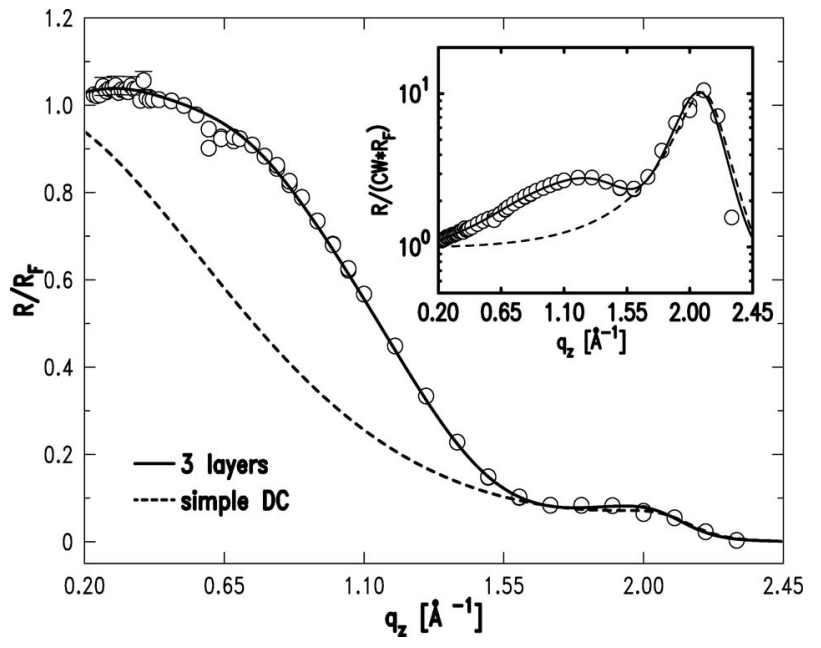

FIG. 3. The background subtracted $\mathrm{x}$-ray reflectivity, $R / R_{F}$ and the one calculated from the best fit of the electron density model given by Eq. (3). The dashed line is the fit from the simplest DC model that is discussed in the text. The inset illustrates the same data after division by $C W\left(q_{z}, T\right)$ along with the fits from the corresponding models.

$$
\Phi\left(q_{z}\right)=\frac{1}{\rho_{\infty}} \int d z \frac{d\langle\rho(z)\rangle}{d z} \exp \left(i q_{z} z\right)
$$

where $\langle\rho(z)\rangle$ is the thermal average of the surface-normal electron density over the illuminated portion of the surface at a vertical position $\mathrm{z}$ and $\rho_{\infty}$ is the electron density in the bulk liquid. Since the form of $R_{F}\left(q_{z}\right)$ is determined completely by the critical angle for total external reflection, and $C W\left(q_{z}, T\right)$ is known accurately from capillary wave theory, the intrinsic electron density profile, $\langle\rho(z)\rangle$, can be obtained by numerically curve-fitting the measured $R\left(q_{z}\right)$ to a physically motivated model. ${ }^{10,11}$

The distorted crystal (DC) model provides a very good description of the single surface-layering peak in $\mathrm{x}$-ray reflectivity at $q_{z} \approx \pi / a$ (a: atomic radius) that is observed for many elemental liquid metals. ${ }^{16-18,23}$ The simplest form of the model for the electron density consists of a series of Gaussian peaks centered at $z_{n}=d_{0}(n-1)$ for $n=1,2,3, \ldots$. In the DC model the Gaussians have common integrated densities, and widths that increase linearly with distance from the surface. For example, if the width of the Gaussian peak in the $n$th layer is given by $\sigma_{n}^{2}=\sigma_{0}^{2}+(n-1) \bar{\sigma}^{2}$ these Gaussian peaks become broader and merge to represent the bulk density at large $n$. This model naturally describes the decay of surface induced layering with distance from the surface. Furthermore, this model has the advantage that the Fourier transform of Eq. (2), can be computed analytically. The Fourier transform of this series of equally-spaced Gaussian peaks gives rise to the surface-layering peak that is observed in the reflectivity. Additional features, other than a simple surfacelayering peak, that are observed in binary liquid metal alloys Bi-In (Ref. 10) and Bi-Sn (Ref. 11) can be described by modifying either the integrated densities in individual layers or the relative position of the top few layers.

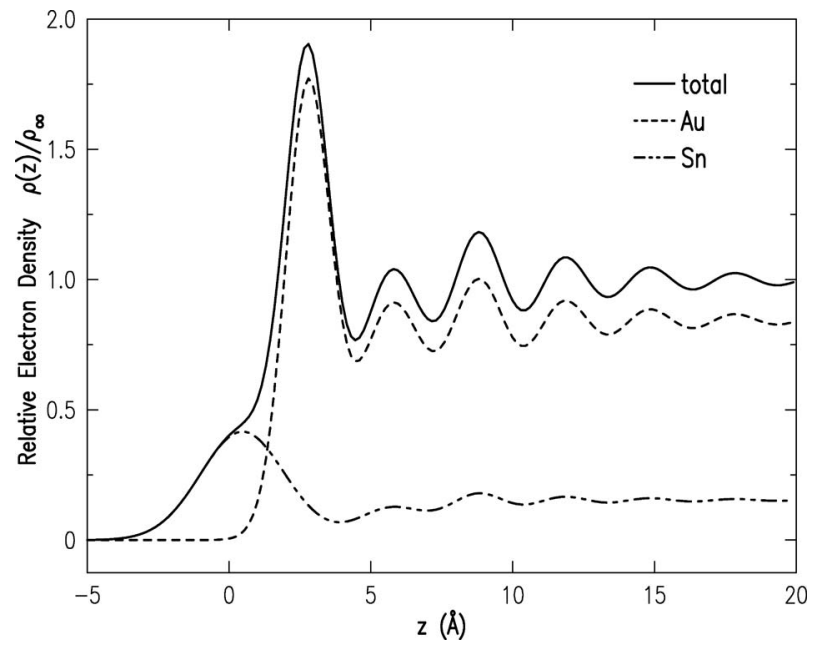

FIG. 4. The surface-normal electron density profile of the AuSn liquid alloy surface as obtained from the best fit to the density model, Eq. (3). of the reflectivity data. The atomic densities of Au and $\mathrm{Sn}$ that contribute to the total density are also shown.

The modified electron density model that is used here to describe the observed dependence on $q_{z}$ of the x-ray reflectivity from the $\mathrm{Au}_{71} \mathrm{Sn}_{29}$ liquid alloy surface differs from the standard DC model in that it treats amplitudes, positions and roughness for the first three layers as independent parameters. The form for the modified model is

$$
\frac{\langle\rho(z)\rangle}{\rho_{\infty}}=\sum_{n=1}^{\infty} \frac{e^{-\left(z-z_{n}\right)^{2} / \sigma_{n}^{2}}}{\sqrt{2 \pi} \sigma_{n} / d_{0}}\left(\left(1-x_{n}\right) \rho_{\mathrm{Au}}^{r e l}+x_{n} \rho_{\mathrm{Sn}}^{r e l}\right) \frac{C_{n}}{C}
$$

where the positions of the first three layers, $z_{1}, z_{2}$, and $z_{3}$, and their widths, $\sigma_{1}, \sigma_{2}$, and $\sigma_{3}$ are adjustable parameters. For $n \geqslant 4$ the layer positions and widths of the Gaussians are $z_{n}$ $=z_{3}+(n-3) d_{0}$ and $\sigma_{n}^{2}=\sigma_{0}^{2}+(n-1) \bar{\sigma}^{2}$. The amplitudes of the Gaussians are expressed in terms of effective x-ray energy dependent electron densities of $\mathrm{Au}$ and $\mathrm{Sn}$ atoms, $\rho_{\mathrm{Au}}^{r e l}, \rho_{\mathrm{Sn}}^{r e l}$, relative to the bulk alloy. They are obtained from the real part of the atomic scattering factors, $Z^{\prime}(E) .{ }^{10,11}$ The concentration of $\mathrm{Sn}$ atoms in the $n$th layer is $x_{n}$ and $C_{n}\left[x_{n} v_{\mathrm{Sn}}+(1\right.$ $\left.\left.-x_{n}\right) v_{\mathrm{Au}}\right]=1$ for the $n$th layer and $C\left[x_{\infty} v_{\mathrm{Sn}}+\left(1-x_{\infty}\right) v_{\mathrm{Au}}\right]=1$ for the bulk, where $C$ and $C_{n}$ are the average atomic densities in the bulk and $n$th layer respectively, and $v_{\mathrm{Sn}}, v_{\mathrm{Au}}$ are the atomic volumes of $\mathrm{Sn}$ and $\mathrm{Au}$ atoms. Neglecting the effect of the imaginary part of the atomic scattering amplitude, the scattering can be described in terms of the forward atomicscattering factor of the atoms. For $\mathrm{Au}$ this is given by, $Z_{\mathrm{Au}}^{\prime}(E)=Z_{\mathrm{Au}}+f^{\prime}{ }_{\mathrm{Au}}(E)$ (Ref. 22) and

$$
\rho_{\mathrm{Au}}^{r e l}=n_{\mathrm{Au}} Z^{\prime}{ }_{\mathrm{Au}}(E) / \rho_{\infty}
$$

where $n_{\mathrm{Au}}$ is the number density of $\mathrm{Au}$ atoms and $f_{\mathrm{Au}}^{\prime}(E)$ is the dispersive correction to the real part of the atomic scattering amplitude. In principle, a similar expression describes the effective relative electron density for $\mathrm{Sn}$; however, the present experiment is done near the Au L2 edge for which the dispersion correction to $Z_{S n}^{\prime}(E)$ is negligible.

The background subtracted Fresnel-normalized reflectivity, $R\left(q_{z}\right) / R_{F}\left(q_{z}\right)$, at the $\mathrm{x}$-ray energy of $13.15 \mathrm{keV}$ is shown 
TABLE I. The best fit parameters from curve fitting the reflectivity data to the modified distorted crystal model discussed in the text. The Sn concentrations $\left(x_{1}, x_{2}, x_{3}\right)$ of the top three modified layers that deviate from the bulk concentration along with the confidence limits, the positions of these layers $\left(z_{1}, z_{2}, z_{3}\right)$ and their interfacial roughnesses $\left(\sigma_{1}, \sigma_{2}, \sigma_{3}\right)$ are given. The layer spacing $\left(d_{0}\right)$ for layers beyond $n=3$ is also given along with the roughness parameters that determine the roughness of these layers, $\sigma_{0}$ and $\bar{\sigma}$.

\begin{tabular}{lcc}
\hline \hline & $\begin{array}{c}\text { Positions and } \\
\text { spacings }(\AA) \text { of } \\
\text { top layer and } \\
\text { others below }\end{array}$ & Roughness $(\AA)$ \\
\hline$x_{1}=0.958(0.957, \cdots)^{\mathrm{a}}$ & $z_{1}=0.47 \pm 0.01$ & $\sigma_{1}=1.45$ \\
$x_{2}=0.006(\cdots, 0.007)^{\mathrm{a}}$ & $z_{2}=2.78 \pm 0.01$ & $\sigma_{2}=0.764$ \\
$x_{3}=0.239(0.0,0.29)$ & $z_{3}=5.75 \pm 0.01$ & $\sigma_{3}=1.161$ \\
$n>3$ & $d_{0}=2.93$ & $\sigma_{0}=0.525$ \\
& & $\bar{\sigma}=0.514$ \\
\hline \hline
\end{tabular}

aThe upper and lower confidence limits of $x_{1}$ and $x_{2}$, respectively, lie outside the range of the allowed values $x_{1}$ and $x_{2}$ can take, namely, 0 to 1 .

in Fig. 3. The inset shows the surface structure factor, $\left|\Phi\left(q_{z}\right)\right|^{2}$, which is the Fresnel-normalized data, further normalized by division with the background-subtracted DebyeWaller term. The measured $R\left(q_{z}\right) / R_{F}\left(q_{z}\right)$ in the $q_{z}<1.6 \AA^{-1}$ region, is strongly enhanced over the prediction from the DC model (dashed line). The simple DC model for a layered interface with uniform composition that has been used for $\mathrm{Ga},{ }^{18} \mathrm{In},{ }^{16}$ and $\mathrm{K},{ }^{17}$ predicts the surface-induced layering peak at $q_{z} \sim 2.0 \AA^{-1}$ but can not produce the broad hump centered at $q_{z} \sim 1.1 \AA^{-1}$. This feature is an unambiguous indication that either the structure or chemical composition of the near surface region deviates from the DC model.

The best representation of the measured reflectivity in Fig. 3 is obtained by numerical fitting of the surface structure factor to the density model given by the real space density $\left\langle\rho(z) / \rho_{\infty}\right\rangle$ in Eq. (3). As was mentioned above, the simple DC model that has the same integrated electron density for each layer cannot explain the behavior of the surface structure factor. The electron density of the top few layers had to be modified to fit the data. In many respects this is similar to the case of BiSn liquid alloy that was studied earlier. ${ }^{11}$ However, even after allowing for the deviation of the electron density and consequently the composition of the top three layers, the data could not be fit satisfactorily. The acceptable fits shown in Fig. 3 were only obtained after allowing the amplitudes, widths, and the spacing of these three layers to be modified. As expected from the Gibbs theory the top layer should be rich in the low surface-tension component, Sn and the model obtains an integrated electron density in the first layer that is definitely lower than for any comparable region in the bulk. This corresponds to a Sn rich first layer. The best fits for the second layer also reveals an unambiguously Aurich region. (Fig. 4, Table I). One word of caution about the meaning of the values in Table I. is that since the widths of the individual Gaussians are comparable to their spacings the concentrations $x_{1}, x_{2}$, and $x_{3}$ should not be interpreted liter- ally as the concentrations in well defined layers and the electron density profiles shown in Fig. 4. are the only realistic representation of the atomic distributions. Nevertheless, the fits do give some information on the atomic concentrations in the third layer. The best fit shown in Fig. 4. shows a peak density in the region of the third layer that is less than that of the fourth layer and an average electron density that is less than the bulk value. On the other hand, the Sn to Au fraction at the peak of the electron density at the position of the third layer does correspond to the value of $x_{3}$ in Table I. This value is less than the concentration for the bulk alloy, implying that this layer is slightly enriched in Au. In view of the fact that the bulk alloy, $\mathrm{Au}_{71} \mathrm{Sn}_{29}$, has slightly more than twice as much $\mathrm{Au}$ than $\mathrm{Sn}$ it may not be surprising to find some $\mathrm{Au}$ enrichment in the layer following monolayers of Sn and Au.

\section{SURFACE-SEGREGATION PROFILES AND STATISTICAL THERMODYNAMICS CALCULATIONS}

It has been well known for many years that surface segregation in binary liquid alloys occurs due to the different surface tensions of the constituent atoms in their respective liquid state. In general the surface energy of the alloy is reduced by surface adsorption of the element with the lower surface tension. ${ }^{1}$ However, it is only recently that x-ray measurements have yielded the type of data, as shown here, that allow quantitative comparison between theoretical models for the details in how the constituent atoms distribute themselves over the different near surface layers. For example, the surface profiles of BiIn (Ref. 10) and BiSn (Ref. 11) have been quantitatively accounted for by calculations based on the solution theories of Guggenheim ${ }^{24}$ and its extension by Defay and Prigogine ${ }^{25}$ and also by Strohl and King. ${ }^{26}$ These theories assume a lattice model and treat the binary liquid as either an ideal or a regular solution to account for the composition of the surface-segregated layers using only the surface tensions of the pure components, their atomic radii, and the interaction energy of the components in the case of regular solutions. In contrast for both the BiIn and BiSn systems the near surface layers are relatively well defined and representation of the surface layering with a lattice model is probably better than for the AuSn alloy. Nevertheless, it is interesting to examine the predictions of these models for the current alloy.

Guggenheim assumed a regular solution model and, taking a statistical thermodynamic approach, developed (Eq. (4)) to predict the surface segregation in the top monolayer in terms of the surface concentration and interaction energy $w$. The surface tension of the alloy in Guggenheim's theory can be represented as

$$
\begin{aligned}
\gamma_{\mathrm{Sn}-\mathrm{Au}}= & \gamma_{\mathrm{Sn}}+\frac{k T}{A_{\mathrm{Sn}}} \ln \left(\frac{x_{1}}{x_{\infty}}\right)+\frac{w}{A_{\mathrm{Sn}}}\left[l\left(1-x_{1}\right)^{2}-(l+m)(1\right. \\
& \left.\left.-x_{\infty}\right)^{2}\right]=\gamma_{\mathrm{Au}}+\frac{k T}{A_{\mathrm{Au}}} \ln \left(\frac{1-x_{1}}{1-x_{\infty}}\right)+\frac{w}{A_{\mathrm{Au}}}\left[l x_{1}^{2}-(l\right. \\
& \left.+m) x_{\infty}^{2}\right]
\end{aligned}
$$


where $x_{1}\left(1-x_{1}\right)$ is the surface concentration in the uppermost layer of component $\mathrm{Sn}(\mathrm{Au})$, and the corresponding concentrations in the bulk are $x_{\infty}\left(1-x_{\infty}\right)$. The surface tensions $\gamma_{\mathrm{Sn}}$ and $\gamma_{\mathrm{Au}}$ correspond to those of pure liquid Sn and Au extrapolated to the measurement temperature of x-ray reflectivity, $T=568 \mathrm{~K}, \gamma_{\mathrm{Sn}}=559 \mathrm{mN} / \mathrm{m}$, and $\gamma_{\mathrm{Au}}=1258 \mathrm{mN} / \mathrm{m} .^{27}$ The interaction energy, $w$ is given by, $w=2 E_{A B}-\left(E_{A A}+E_{B B}\right)$ where the $E_{A A}, E_{B B}$, and $E_{A B}$ are the different types of atomic interaction energies; however, since the interaction energies are not well known, $w$ is taken to be an adjustable fitting parameter. The atomic areas, $A_{\mathrm{Sn}}$ and $A_{\mathrm{Au}}$ have been calculated from the atomic radii of the two components, $a_{\mathrm{Sn}}$ $=1.41 \AA$ and $a_{\mathrm{Au}}=1.44 \AA$ (Ref. 28) and $l$ and $m$ are the (fractional, $l+2 m=1$ ) lateral and vertical coordination numbers. Their values were assumed to be 0.5 and 0.25 that correspond to those of a hexagonally close-packed lattice with 12 nearest neighbors. With the atomic radii, the hexagonally close-packed coordination numbers, the temperature and the bulk alloy composition $x$ known, the only unknown parameters in Eq. (4) are $x_{1}, w$, and $\gamma_{\mathrm{Sn}-\mathrm{Au}}$. Equating the left and right hand sides, leaves only $x_{1}$ and $w$ unknown. In principle, one could solve either for $x_{1}$ for a given value of $w$, or solve for $w$ for a given value of $x_{1}$ to obtain a predicted surface tension.

Neglecting interactions (i.e., $w=0$ ) and assuming an ideal solution behavior for the AuSn liquid alloy, the calculated concentration of the top most layer turns out to be $0.992 \mathrm{Sn}$, that is almost a pure monolayer of Sn. While this Sn concentration is close to the experimental value of 0.958 obtained from the curve-fitting analysis of the x-ray reflectivity data, Eq. (4), yields a surface tension of $713 \mathrm{mN} / \mathrm{m}$ that is $16 \%$ larger than the experimental value of $615 \mathrm{mN} / \mathrm{m}$. On the other hand, the experimental observation of nonzero enthalpy of mixing for the Au-Sn alloys ${ }^{14}$ suggests that the interactions between atoms cannot be neglected. Since we are interested in understanding whether the observed composition of the surface layer $x_{1}$ can explain the surface tension we fixed $x_{1}=0.958$ and solved (Eq. (4)) for the interaction energy, $w=-2.20 \mathrm{kT}$. (The value changes to $w=-2.23 \mathrm{kT}$ if $x_{1}$ is set at the lower confidence limit 0.957). Unfortunately, the surface tension obtained with finite $w$ yields an even larger value, $876 \mathrm{mN} / \mathrm{m}$. These results are summarized in the second and third columns of Table II.

In view of the fact that Guggenheim's theory does not include effects associated with concentration variations beyond the top layer we considered the possibility that the discrepancy in the surface tension might be resolved by inclusion of effects associated with the observed deviation of the concentration in the second and third layers from the bulk values (Fig. 4). The Defay-Prigogine (D-P) modification of the Guggenheim theory has been applied successfully to explain the surface-segregation in a BiSn liquid alloy ${ }^{11,25}$ and, in spite of the already mentioned fact that the layers are not as well defined as for the BiSn system we examined the D-P prediction. In this model the Sn-concentration, $x_{2}$, of the second layer can be calculated from that of the first layer, $x_{1}$, using Eq. (5) (Refs. 11 and 25)
TABLE II. The concentrations and surface tension calculated from the Guggenheim and Defay-Prigogine (DP) as discussed in the text. The interaction parameter $w / \mathrm{kT}=-2.20$ that was derived from the Guggenheim theory was used for the D-P theory along with the experimental value for $x_{1}$. The Sn concentrations in the brackets correspond to the interaction energy $w / \mathrm{kT}=-2.23$ calculated from the lower confidence limit of $x_{1}=0.957$.

\begin{tabular}{lllcc}
\hline \hline Parameters & \multicolumn{2}{c}{ Guggenheim } & D-P & Experimental values \\
\hline$w / \mathrm{kT}$ & 0 & & -2.20 & \\
$x_{1}$ & 0.9908 & $0.958^{\mathrm{a}}$ & $0.958^{\mathrm{a}}$ & $0.958(0.957, \cdots)$ \\
$x_{2}$ & & $\cdots$ & $0.195(0.194)$ & $0.006(\cdots, 0.007)$ \\
$x_{3}$ & & $\cdots$ & $0.305(0.305)$ & $0.239(0.0,0.29)$ \\
$x_{4}$ & & & $0.287(0.288)$ & 0.29 \\
$x_{\infty}$ & & & & 0.29 \\
$\gamma(\mathrm{mN} / \mathrm{m})$ & 713 & 815 & 876 & $615(600,640)$ \\
\hline \hline
\end{tabular}

axperimental value.

$$
\begin{aligned}
& \ln \left(\frac{\left(1-x_{2}\right) x_{\infty}}{\left(1-x_{\infty}\right) x_{2}}\right)-\frac{2 w}{k T}\left[(1-2 m)\left(1-x_{2}\right)-\left(1-x_{\infty}\right)\right] \\
& \quad=\frac{2 w m}{k T}\left(2-x_{\infty}+x_{1}\right)
\end{aligned}
$$

The linearized D-P prediction for the correction to the surface tension is given by

$$
\gamma_{D-P}=\frac{2 m w}{\bar{A}}\left(1-x_{\infty}\right)\left(x_{\infty}-x_{1}\right)+\gamma_{G u g g} .
$$

In this modification, where $\bar{A}$ is the average atomic area for $\mathrm{Au}_{71} \mathrm{Sn}_{29}$, the interaction parameter and/or the value of $x_{1}$ is unchanged from values obtained from the Guggenheim theory (Eq. (4)). Although the Sn concentrations in the third and fourth layers can be calculated from the concentration in the second and third layers, respectively, the surface tension correction to the linearized D-P theory only involves the concentration in the first layer, $x_{1}$. The results from the D-P equations with the Guggenheim values of $w / \mathrm{kT}=-2.20$ and $x_{1}=0.958$ are shown in the fourth column of Table II.

The agreement with experiment is at best only qualitative. The first problem is, as already explained, the association between the concentrations in the layered models of Guggenheim and Defay-Prigogine with the Gaussian amplitudes of the distorted crystal model is not strict. It is encouraging that both models predict surface layers that are essentially pure $\mathrm{Sn}$, in agreement with the experimental best fit. On the other hand, it is hard to know how one should interpret the discrepancy between the nearly zero concentration of the $\mathrm{Sn}$ in the second layer of the distorted crystal model and the $20 \mathrm{~atm} . \%$. Sn concentration in the second layer of the D-P model. For example, the tail of the Gaussian peak of distorted crystal model that represents the first layer of Sn partially overlaps the Gaussian peak that represents the second layer that is $\mathrm{Au}$. For these Gaussians the ratio of the $\mathrm{Sn}$ to $\mathrm{Au}$ density at the peak position of the Au Gaussian, $z=2.78$, corresponds to a Sn concentration of nearly $30 \%$ that is not 
TABLE III. The Sn concentrations calculated from S-K by optimization of $w / \mathrm{kT}=-1.78$. The third column lists the values calculated from D-P with the interaction parameter that was obtained from S-K theory.

\begin{tabular}{llll}
\hline \hline Parameters & \multicolumn{1}{c}{$\mathrm{S}-\mathrm{K}$} & $\mathrm{D}-\mathrm{P}\left({ }^{*}\right)$ & \multicolumn{1}{c}{ Experimental } \\
\hline$x_{1}$ & 0.995 & 0.969 & $0.958(0.957, \cdots)$ \\
$x_{2}$ & 0.009 & 0.206 & $0.006(\cdots, 0.007)$ \\
$x_{3}$ & 0.309 & 0.301 & $0.239(0.0,0.29)$ \\
$x_{4}$ & 0.287 & 0.288 & 0.29 \\
$\gamma(\mathrm{mN} / \mathrm{m})$ & $\cdots$ & 847 & 615 \\
\hline \hline
\end{tabular}

so different from the $20 \%$ of the D-P model. On the other hand there seems to be a clear disagreement between the D-P prediction for a third layer that is slightly enriched in $\mathrm{Sn}$ and the result from the distorted crystal model in which the third layer is enhanced in Au.

Strohl-King (S-K) have developed an alternative to the $\mathrm{D}-\mathrm{P}$ theory which unfortunately has the same shortcoming of assuming well defined layers. ${ }^{26}$ This theory can be used to estimate the concentrations in the various layers by an iterative procedure in which the concentrations in the various layers can be adjusted to match the measured concentrations by varying the interaction parameter. The basic equation for the concentrations is

$$
\begin{aligned}
1-x_{n}= & \left(1-x_{\infty}\right) \exp \left(\frac { w } { k T } \left\{x_{\infty}^{2}-\left(\frac{l x_{n}^{2}}{l+m}\right)+\left(\frac{m}{l+m}\right)\left[\left(x_{n-1}\right)^{2}\right.\right.\right. \\
& \left.\left.\left.+\left(x_{n+1}\right)^{2}\right]\right\}+\frac{A_{\mathrm{Au}}\left(\gamma_{\mathrm{Sn}}-\gamma_{\mathrm{Au}}\right) x_{n}}{k T}\right) .
\end{aligned}
$$

The calculated values that are shown in the second column of (Table III) correspond to the value of the interaction parameter $w / \mathrm{kT}$ that is obtained by minimization of $\chi^{2}$ $\equiv \sum_{n=1}^{10}\left(x_{n}-x_{n}^{\mathrm{exp}}\right)^{2}$. The optimum interaction energy $w=$ $-1.78 \mathrm{kT}$ is about $20 \%$ lower than the D-P value of $-2.2 \mathrm{kT}$. Although the S-K theory does predict a near vanishing of the Sn concentration in the second layer it is hard to know the significance of this. For completeness the values in the third column of Table III. demonstrate that the results from D-P for $w / \mathrm{kT}=-1.78$ (Table III) are not significantly different from the D-P values shown in Table II for $w / \mathrm{kT}=-2.2$ that were obtained from the Guggenheim equation (Eq. (4)) when $x_{1}$ was fixed at the experimental concentration.

Various thermophysical properties such as the enthalpy of mixing, surface tension, viscosity and the surface compositions of the liquid AuSn alloy have been calculated recently by Novakovic et al. ${ }^{20}$ using what they refer to as a "complex formation model" that is a combination of Guggenheim and other statistical-thermodynamic theories. Although the surface-concentration of $\mathrm{Sn}$ they obtain $(\sim 0.65)$ does exceed the bulk value, it is still significantly smaller than the value extracted from the $\mathrm{x}$-ray reflectivity data. Similarly, for 29 atm. \% Sn their surface tension $(\sim 960 \mathrm{mN} / \mathrm{m})$ is also much larger than the X-ray determined value of $615 \mathrm{mN} / \mathrm{m}$. On the other hand, the interaction energy parameters, used to calculate the surface concentration profiles, -2.42 (at
$550{ }^{\circ} \mathrm{C}$ ) and $-1.25\left(\right.$ at $1100{ }^{\circ} \mathrm{C}$ ) extrapolate linearly to -3.0 at $300{ }^{\circ} \mathrm{C}$, that is only $\sim 25 \%$ larger than the value of -2.2 obtained from Guggenheim and D-P theories. This can be compared to bulk interaction energy $w_{\text {bulk }}=\Delta H_{m} / x(1-x)$ $=-9.98 \mathrm{kT}$ that is calculated from the enthalpy of mixing, $\Delta H_{m}=-9.66 \mathrm{~kJ} / \mathrm{mole}$, which is nearly a factor of four larger than the surface interaction energy obtained here. ${ }^{14}$ In fact, a similar disagreement was observed for $\mathrm{SnBi},{ }^{11}$ where the argument was made that that bulk thermodynamic quantities often yield inaccurate values for surface quantities.

\section{CONCLUSIONS}

X-ray reflectivity studies show that the atomic-level surface segregation occurs in the eutectic AuSn liquid alloy and extends up to three layers. The top layer is almost a pure monolayer of the low surface-tension component, Sn. The extent of segregation observed is similar to that found in BiSn alloys where the surface-segregation probably extends up to three layers with the top most layer very rich in the low-surface tension component, $\mathrm{Bi}$. The $\mathrm{Au}_{71} \mathrm{Sn}_{29}$ system differs from the nearly equimolar $\mathrm{Bi}_{43} \mathrm{Sn}_{57}$ in that for the latter the enrichment by layers was $\mathrm{Bi}: \mathrm{Sn}: \mathrm{Bi}$ while here it appears to be Sn:Au:Au. The extent of segregation observed is more pronounced than in BiIn that also has negative enthalpy of mixing. All of the liquid alloys studied so far by $\mathrm{x}$-ray reflectivity show the segregation of the low surfacetension component to the surface independent of whether their enthalpy of mixing in the bulk is positive or negative. This behavior shows that the surface energy plays a dominant role in surface segregation. While the simple theories of surface segregation can qualitatively explain the surface segregation profiles reasonably well, they cannot account for the measured surface tension and the agreement regarding concentrations is not quantitative. For the AuSn liquid alloy studied here the existing theories cannot satisfactorily explain both the surface segregation profiles and the surface tension together. There clearly is a need for improvements in the theory of the surfaces of liquid metal alloys.

Finally we should point out that equally good fits to the reflectivity can be obtained by a different model in which the top surface layer is Au-rich rather than $\mathrm{Sn}$ rich. This is not completely surprising in that it is known that the lack of phase information due to the fact that the reflectivity depends on the absolute value of the surface structure factor implies an ambiguity in interpretation. ${ }^{29}$ Fortunately, the physical constraint imposed by the Gibbs adsorption allows the Aurich surface model to be dismissed. Aside from this the Guggenheim, and Defay-Prigogine theories would require an interaction energy parameter of $72(w / k T)$ in order to explain the Au rich surface composition model. Even after allowing for the above mentioned uncertainty relating bulk and surface quantities, this very large positive interaction parameter would imply a very large positive enthalpy of mixing that is not observed in the bulk and is certainly not physically realistic.

\section{ACKNOWLEDGMENTS}

This work has been supported by the U.S. Department of 
Energy through Grant No. DE-FG02-88-ER45379, ChemMatCARS is principally supported by the National Science Foundation/Department of Energy under Grant No.
CHE0087817. The Advanced Photon Source is supported by the U.S. Department of Energy, Basic Energy Sciences, Office of Science, under Contract No: W-31-109-Eng-38.
${ }^{1}$ J. W. Gibbs, The collected works of J. Willard Gibbs (Longmans, Green and Co., New York, 1928).

${ }^{2}$ A. W. Adamson and A. P. Gast, Physical Chemistry of Surfaces (John Wiley \& Sons Inc, New York, 1997).

${ }^{3}$ A. B. Bhatia and N. H. March, J. Chem. Phys. 68, 4651 (1978).

${ }^{4}$ K. N. Tu, A. M. Gusak, and M. Li, J. Appl. Phys. 93, 1335 (2003).

${ }^{5}$ J.-H. Kuang, M.-T. Sheen, C.-F. H. Chang, C.-C. Chen, G.-L. Wang, and W.-H. Cheng, IEEE Trans. Adv. Packag. 24, 563 (2001)

${ }^{6}$ J. G. Lee and H. Mori, Phys. Rev. B 70, 144105 (2004).

${ }^{7}$ H. Yasuda, K. Mitsuishi, and H. Mori, Phys. Rev. B 64, 094101 (2001).

${ }^{8}$ P. Huber, O. G. Shpyrko, P. S. Pershan, B. M. Ocko, H. Tostmann, E. DiMasi, and M. Deutsch, Phys. Rev. Lett. 89, 035502 (2002)

${ }^{9}$ P. Huber, O. G. Shpyrko, P. S. Pershan, B. Ocko, E. DiMasi, and M. Deutsch, Phys. Rev. B 68, 085401 (2003).

${ }^{10}$ E. DiMasi, H. Tostmann, O. G. Shpyrko, P. Huber, B. M. Ocko, P. S. Pershan, M. Deutsch, and L. E. Berman, Phys. Rev. Lett. 86, 1538 (2001).

${ }^{11}$ O. G. Shpyrko, A. Y. Grigoriev, R. Streitel, D. Pontoni, P. S. Pershan, M. Deutsch, B. Ocko, M. Meron, and B. H. Lin, Phys. Rev. Lett. 95, 106103 (2005).

${ }^{12}$ D. Zivkovic, D. Manasijevic, and Z. Zivkovic, J. Therm Anal. Calorim. 79, 71 (2005).

${ }^{13}$ H. Tostmann, E. DiMasi, O. G. Shpyrko, P. S. Pershan, B. M. Ocko, and M. Deutsch, Phys. Rev. Lett. 84, 4385 (2000).
${ }^{14}$ R. Hultgren, R. L. Orr, P. D. Anderson, and K. K. Kelley, Selected Values of the Thermodynamic Properties of Metals and Alloys (Metals Park, OH, 1963).

${ }^{15}$ Active vibration isolation feedback system from Halcyonics Gmbh, Goettingen, Germany.

${ }^{16}$ H. Tostmann, E. DiMasi, P. S. Pershan, B. M. Ocko, O. G. Shpyrko, and M. Deutsch, Phys. Rev. B 59, 783 (1999).

${ }^{17}$ O. G. Shpyrko, P. Huber, A. Y. Grigoriev, P. S. Pershan, B. Ocko, H. Tostmann, and M. Deutsch, Phys. Rev. B 67, 115405 (2003).

${ }^{18}$ M. J. Regan, E. H. Kawamoto, S. Lee, P. S. Pershan, N. Maskil, M. Deutsch, O. M. Magnussen, B. M. Ocko, and L. E. Berman, Phys. Rev. Lett. 75, 2498 (1995).

${ }^{19}$ O. G. Shpyrko, M. Fukuto, P. S. Pershan, B. Ocko, I. Kuzmenko, T. Gog, and M. Deutsch, Phys. Rev. B 69, 245423 (2004).

${ }^{20}$ R. Novakovic, E. Ricci, F. Gnecco, D. Giuranno, and G. Borzone, Surf. Sci. 599, 230 (2005).

${ }^{21}$ P. S. Pershan and J. Als-Nielsen, Phys. Rev. Lett. 52, 759 (1984).

${ }^{22} \mathrm{~J}$. Als-Nielsen and D. McMorrow, Elements of Modern X-ray Physics (Wiley, New York, 2001).

${ }^{23}$ O. M. Magnussen, B. M. Ocko, M. J. Regan, K. Penanen, P. S. Pershan, and M. Deutsch, Phys. Rev. Lett. 74, 4444 (1995).

${ }^{24}$ E. A. Guggenheim, Trans. Faraday Soc. 41, 150 (1945).

${ }^{25}$ R. Defay and I. Prigogine, Trans. Faraday Soc. 46, 199 (1950).

${ }^{26}$ J. K. Strohl and T. S. King, J. Catal. 118, 53 (1989).

${ }^{27}$ T. Iida and R. I. L. Guthrie, The Physical Properties of Liquid Metals (Clarendon Press, Oxford, 1993).

${ }^{28}$ J. Emsley, The Elements (Clarendon Press, Oxford, 1994).

${ }^{29}$ P. S. Pershan, Phys. Rev. E 50, 2369 (1994). 